\title{
Emerging Non-Pharmacological Therapies for Post-stroke Depression and its Future Aspects: A Review
}

\author{
Keerthana Chandrasekar, Nakka Gautam Sai, Princy Sabu John, Sruthi Ninan, Raja Durai, \\ Sivasankaran Ponnusankar*
}

Department of Pharmacy Practice, JSS College of Pharmacy, JSS Academy of Higher Education and Research, Ooty, Tamil Nadu, INDIA.

\begin{abstract}
Post-Stroke Depression (PSD) is a psychiatric disorder associated with stroke which has an adverse effect on the cognitive function and survival. It usually develops in $40 \%$ of the stroke survivors within 3 months. We performed a thorough literature review using Psychlnfo, PubMed, Science Direct and PLOS databases for the non-pharmacological treatment of PSD. Early rehabilitation and psychological therapies are effective in treating depression in PSD while physiotherapy and music therapy improves both cognitive and movement disability. Transcranial stimulation and electroconvulsive therapy are invasive procedures used to treat cognitive impairment in PSD. Computerized therapy helps, enhance the attention, memory and executive functioning. DepReT stroke is a package which addresses patients along with their caregiver to have a better understanding of the depression. Robot assisted rehabilitation along with traditional rehabilitation is effective in treating motor function associated with PSD. Early assessment, treatment and rehabilitation are the most effective ways to prevent depression in stroke survivors and thereby improving their quality of life. Non-pharmacological treatment for post-stroke depression seem to be efficacious as antidepressants in improving depressive symptoms with minimal side effects.
\end{abstract}

Key words: Caregiver, Cognitive Impairment, Non-Pharmacological Treatment, PostStroke Depression, Quality of Life, Rehabilitation.

\section{INTRODUCTION}

Stroke is defined as the sudden death of brain cells due to reduced blood supply to the brain usually caused by thrombotic, embolic and other hemorrhagic events. ${ }^{1}$ Post-stroke depression (PSD) is a mood disorder occurring after stroke and moreover, an important neuropsychiatric consequence of stroke. $^{2} 40 \%$ of stroke survivors will proceed to post-stroke depression within 3 months. ${ }^{3}$

Depletion of neurotransmitter biogenic amine and changes in post synaptic receptor sensitivities play an important role in the development of post stroke depression. ${ }^{4}$ On an average of 10 years, the mortality rate amongst post-stroke depressive patients is
3.4 times which indicates the need of treatment in post-stroke patients. ${ }^{3}$

Pharmacological therapies such as selective serotonin reuptake inhibitor and tricyclic antidepressants are effective in the treatment of post-stroke depression, but these drugs have well known side effects. ${ }^{5}$ An analysis of a cohort of patients with stroke, proved that there is a greater risk of stroke $(48 \%)$ associated with anti-depressants. ${ }^{6}$ This article discusses about the various nonpharmacological treatments recommended for post-stroke depression, which are as efficacious as pharmacological therapy with minimal side effects.
Submission Date: 02-08-2018; Revision Date: 17-07-2019; Accepted Date: 06-11-2019

DOI: 10.5530/ijper.54.1.1 Correspondence: Dr. Sivasankaran Ponnusankar Professor and Head, Department of Pharmacy Practice, JSS College of Pharmacy, JSS Academy of Higher Education and Research, Ooty-643001, Tamil Nadu, INDIA.

Phone: +91-0423-2443393 E-mail: ponnusankarsivas@ gmail.com

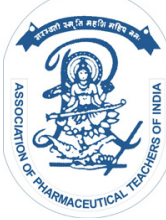

www.ijper.org 


\section{MATERIALS AND METHODS}

We performed a detailed review on the available databases such as PsychInfo, PubMed, ScienceDirect and PLOS databases and articles from 1992 till 2017 in English were reviewed, using search words such as poststroke depression, non-pharmacological treatments, cognitive impairment, quality of life, rehabilitation and caregiver. The literatures were reviewed thoroughly and non-pharmacological therapies are discussed in the article. This review discusses on the evidences of nonpharmacological treatments used to treat post-stroke depression.

\section{RESULTS}

Non-pharmacological therapies are known to be effective in the treatment of post-stroke depression and the emerging non-pharmacological treatments are listed in Figure 1.

\section{Self-Management}

The main goals of self-management are managing their emotional consequences after stroke, managing medical task, to maintain their behaviour and to deal with the psychological consequences of the stroke. The five self-management skills are: problem solving, decision making, proper utilization of the resources, developing a rapport with the physician and taking appropriate actions. $^{7}$ Therefore, self-management is a self-control therapy that helps the patient to manage their depression and self-management skills.

\section{Rehabilitation}

Stroke patients with more than one mental illness an increased probability to be hospitalized or die when compared to patients with no mental illness, ${ }^{8}$ therefore, early rehabilitation reduces the frequency of cognitive decline and hypothymia. ${ }^{9}$ Rehabilitation is a target oriented therapy, which grants patients with tools to manage their own life by reducing physical, mental and social impairment. ${ }^{10}$ The advantages of rehabilitation are improved self-awareness, self-esteem improvement, stress management. ${ }^{11}$ Cardiac rehabilitation is used as a secondary preventive measure for transient ischemic stroke or mild stroke, but is not associated with any emotional improvement. ${ }^{12}$

\section{Psychological Therapy}

Psychological therapy is effective in treating mild and moderate depression as well as normal and slightly diminished cognitive abilities provided by the trained professionals and general physicians. ${ }^{13}$ Psychosocial intervention is more effective in 5-HTTLPR s-allele carriers and ST in 2 VNTR 9 allele carriers which are functional length polymorphism of the SERT gene. ${ }^{14}$ The psychological therapy includes:

a) Cognitive therapy: Aaron T. Beck discovered cognitive therapy for the first time to treat depression. According to him, if negative thoughts and beliefs are corrected, depressive symptoms will be improved. ${ }^{15}$ Cognitive Behavioural Therapy (CBT) increases the daily activities, especially pleasant and enjoyable events to improve mood and is efficient to treat depression in primary care settings. Behavioural therapy in Behavioural Activation therapy for Depression After Stroke (BEADS) includes active monitoring, activity scheduling, graded task assignment. ${ }^{16}$ Behavioural treatment facilitates problem solving skills and cognitive restructuring skills to manage ongoing tensions. ${ }^{17}$ Augmented cognitive therapy includes various phases and improves to alter negative thoughts, relaxed activities, helps in goal setting and individualized treatment. ${ }^{18,19}$

Problem solving therapy (PST): PST is effective in the treatment of post-stroke depression by reducing mental disability and is mainly efficacious in elderly patients and the effect continues even after the treatment ends. ${ }^{20}$ Consequently, PST reduces the mortality associated with post stroke depression in comparison with escitalopram. ${ }^{21}$

c) Supportive therapy: Supportive therapy is a patient centred psychotherapy. A therapist listens to the patient and provides support based on the patient's problem. Problem solving therapy is more beneficial than supportive therapy in treating mental disability in elderly. ${ }^{20}$

d) Motivational therapy: Motivational therapy motivates the patient to ameliorate medication adherence and follow their lifestyle modifications and thereby creates awareness among the subjects regarding the consequences and hazards as a result of their behaviour

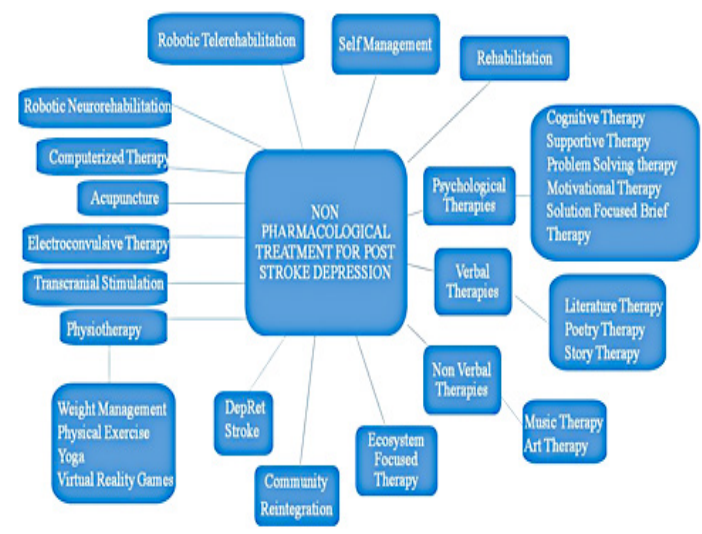

Figure 1: Non pharmacological treatment options to treat PSD. 
and makes them think about their habits and the profit associated with the change in their behaviour or habit. ${ }^{22}$

\section{Verbal Therapy}

Verbal therapy is a psychological therapy for patients with an emotional disability or impairment which involves literature, story and poetry therapy.

a) Literature therapy: Literature therapy is an expressive therapy which serves as a tool to identify the emotional status of the patient and treat emotional and behavioural disorders. ${ }^{23}$

b) Poetry therapy: Poetry therapy improves the intellectual and mental function which helps to lead a normal life by correlating their sentiments to a poet's feelings as it rely upon poem, metaphor to improve the self-awareness and healing. ${ }^{23}$

c) Story therapy: Patients create a story line, in which they wish to live, thereby the story therapy helps in changing the patients depressing state to a bright and fine story. ${ }^{23}$

\section{Non-Verbal Therapy}

Nonverbal therapy is a psychological therapy for patients with language disability or impairment and consists of art and music therapy.

a) Music therapy: Music is beneficial not only does it exert on physiological effects such as blood pressure, heart rate, respiration, but also has an influence on emotional aspects of life and motor functions. ${ }^{24}$ Listening to music generates motions and emotions; a strong bond between realization and action ${ }^{25}$ and additionally enhances both cognitive and emotional functions in healthy people and patients. ${ }^{26} 64.3 \%$ of stroke patients in music therapy showed mood improvement. ${ }^{27}$ Music therapy involves exercises using musical instruments to practice enormous motor function ${ }^{28}$ and consequently, accelerates the motor function by producing a change in the cortical plasticity and rapid plastic adaptation. ${ }^{29}$

b) Art therapy: Art therapy helps the patients to procure the ability to interact within the community, develop a rapport with the therapist and to look into themselves by free self-expression through painting or drawing. Depressive symptoms such as loss of appetite sleep disorders, lack of confidence, over eating is treated by art therapy. ${ }^{30}$

\section{Community Reintegration (CR)}

Community reintegration is the reorganization of physical, psychological and social characteristics of the patient. Assessment of community reintegration is mainly by Reintegration to a Normal Living Index
(RNLI) where it measures the patient's attitude and understanding of their capabilities. ${ }^{31}$

\section{Physiotherapy}

a) Physical exercise: Physical activity consists of voluntary bodily movement that requires energy above resting levels ${ }^{32}$ and in addition, increases dopamine and brain derived neurotropic factor, thereby enhancing mood. ${ }^{33}$ Physical activities play an important role in post-stroke depression by improving functional performance, quality of life and thereby reducing cardiovascular risk and also encourages the stroke survivor. ${ }^{34,35}$

b) Weight management: Weight reduction is effective in post-stroke depression, the latest system CHAN$\mathrm{GE}^{\mathrm{TM}}$ aids to motivate overweight stroke survivors to reduce their weight up to $5 \%$ and to improve their health outcomes, mainly by diet, sleep and physical activity. ${ }^{36}$

c) Virtual reality games: Virtual reality games in addition to standard physiotherapy was found to be as effective as that of standard therapy alone to improve the physical function of the stroke survivor. Virtual games constitutes various tests like Time up and go test, 30 sec sit to stand test, Timed ten metres walk test and Six minutes walk test. ${ }^{37}$

d) Yoga: Yoga extends the health well-being and awareness about oneself and the daily practice of yoga improves plasticity, muscle strength, aerobic capacity and vital capacity and especially for melancholic depression, yoga breathing exercises are beneficial. ${ }^{38}$ No adverse events reported due to yoga practise. ${ }^{39}$

\section{Acupuncture}

Acupuncture therapy (traditional Chinese medicine) was initiated in China, is the process of restoring the neuroimaging activity of the brain region involved in the emotional activity processing and thereby initiating antidepressant effect. ${ }^{40,41}$ Acupuncture when compared to the western medicines in the treatment of post-stroke depression revealed that curative rate is high in acupuncture. ${ }^{42}$ Both fluoxetine and acupuncture improves the symptoms of ischemic post stroke depression, but the latter has no obvious side effects. ${ }^{43}$ The advantages are its low cost and feasibility. ${ }^{44}$

\section{Computerized Therapy}

Cognitive neurorehabilitation, a computerised programme, which employs Schulte's table with biological feedback where the patient has to identify numbers from 1-25 in ascending order within the time provided in training of attention. Noteworthy improvement was observed in cognitive function of the patients who used this computerized cognitive Neurorehabiliation. ${ }^{45}$ 
'Beating the blues' is a computerized cognitive behavioural therapy package to treat the post stroke depression symptoms with best evidence within primary care. ${ }^{46}$

\section{Electroconvulsive Therapy}

Electroconvulsive therapy is a procedure where an electric current is passed through the brain along with the help of an anaesthetic and muscle relaxant which intentionally produces a brief seizure for $20-50 \mathrm{sec}$. Electroconvulsive therapy shows success rate in the treatment for post stroke depression and is well tolerated when the patient offers resistance to pharmacotherapy. ${ }^{47}$

\section{Transcranial Stimulation}

Repetitive transcranial magnetic stimulation is a neuroprotector which targets the distributed brain network responsible for depression and delivers magnetic stimulation in short time. ${ }^{48}$ Cognitive control therapy and transcranial direct current stimulation combination used to treat depressive symptoms in post stroke depression showed a response rate of $25 \% .{ }^{49}$ In conclusion, transcranial stimulation is effective to treat post-stroke depression in patients contraindicated to antidepressant therapy and is beneficial in chronic stroke patients. ${ }^{50}$

\section{Role of Caregivers in Post-Stroke Depression}

Stroke survivors need a carer to support and care for them. However, it shows $40 \%$ of carers will experience depression within 3 months of stroke and furthermore, the patient's impaired motor function and psychological changes will have a great effect on carer's mental health. ${ }^{51}$

DepReT stroke: A manualized intervention, composed of six sessions lasting for $30 \mathrm{~min}$ each, delivered by the nurse where she monitors the medication adherence and ensures that families have a better understanding of the depression. ${ }^{51}$

\section{Future aspects of non-pharmacological treatment in post-stroke depression}

Robot Neurorehabilitation and Robotic Telerehabilitation: Robot assisted rehabilitation, with the help of a robotic instrument can emphasize the improvement of motor function in stroke patients by controlling interaction forces and providing assistance to the patient's disabilities. To conclude, this instrument is efficient in reducing hand impairment and is maintained for 6 weeks after the therapy completion. ${ }^{52}$ Robotic leg orthosis is a portable mobile intention oriented robotic device, battery powered and wearable bionic leg used to improve mobility, gait speed, quality and tolerance. ${ }^{53}$ Robotic Telerehabilitation is the delivery of rehabilitation services and monitoring for the post-stroke depressive patients over telecommunication networks. Telerehabilitation, along with robot assisted therapy, is a novel approach in rehabilitation where the therapist can monitor the patient through telerehabilitation. ${ }^{54}$

\section{DISCUSSION}

Post-stroke depression is a sensitive concern among patients, physicians, caregivers and society because of its psychological, physical and personal consequences. Strangely, more than half of the cases of PSD are neither identified nor treated. Early assessment, treatment and rehabilitation are the most effective way to prevent depression in stroke survivors and thereby improving the quality of life. Pharmacological therapies such as selective serotonin reuptake inhibitors and tricyclic antidepressants are observed to be efficacious in post-stroke depression, however it is associated with greater risk of stroke $(48 \%)$. Non-pharmacological treatment for poststroke depression suggests to be efficacious as antidepressants in improving depressive symptoms, though the former has no obvious side effects and additionally, they are much safer and improve patient's disability, satisfaction, social reintegration, helplessness, motor function, self-esteem, adherence to treatment thereby exhibits better outcomes and quality of life. The emerging and promising non-pharmacological treatments for post-stroke depression are self-management, rehabilitation, cognitive behavioural therapy, supportive therapy, problem solving therapy, motivational therapy, literature therapy, poetry therapy, story therapy, music therapy, art therapy, community reintegration, weight management, physical exercise, yoga, virtual reality games, acupuncture, computerized therapy, electroconvulsive therapy, transcranial stimulation. In the future, non-pharmacological treatment for PSD can play a pivotal role in treating PSD as it is widely acceptable by both patients and caregivers with minimal side effects, when compared to the pharmacological therapies.

\section{CONCLUSION}

Early assessment, treatment and rehabilitation are the most effective way to prevent depression in stroke survivors and thereby improving quality of life. Nonpharmacological treatment for post-stroke depression seems to be efficacious as antidepressants in improving depressive symptoms with minimal side effects.

\section{ACKNOWLEDGEMENT}

The authors would like to thank Ms. Sini Sathish B.Sc. B.Ed. PGD HRM, IELTS British Council certified 
trainer for making necessary language correction to this paper.

\section{CONFLICT OF INTEREST}

The authors of this paper have no conflicts of interest to declare.

\section{ABBREVIATIONS}

PSD: Post Stroke Depression; QOL: Quality of Life; ESD: Education on Stroke and Depression; EFT: Ecosystem Focused Therapy; RNLI: Reintegration to Normal Living Index; PST: Problem Solving Therapy; ECT: Electro convulsive therapy; SERT: Serotonin; TM: Theoretical model; DepReT: Depression Recognition and Treatment; CBT: Cognitive Behavioural Therapy; SFBT: Solution Focussed Behavioural Therapy.

\section{REFERENCES}

1. Robinson RG, Jorge RE. Post-stroke depression: A review. Am J Psychiatry. 2016;173(3):221-31

2. Gall A. Post stroke depression. Hosp Med. 2001;62:268-73.

3. Shi Y, Xiang Y, Yang Y, Zhang N, Wang S, et al. Depression after minor stroke: Prevalence and predictors. J Psychosom Res. 2015;79(2):143-7.

4. Metoki N, Sugawara N, Hagii J, Saito S, Shiroto H, Tomita T, et al. Relationship between the lesion location of acute ischemic stroke and early depressive symptoms in Japanese patients. Ann Gen Psychiatry. 2016;15:12-8.

5. Youn J, Sung K, Song B, Lee S. Effects of Electro-Acupuncture Therapy on Post-Stroke Depression in Patients with Different Degrees of Motor Function Impairments: A Pilot Study. J Phys Ther Sci. 2013;25(6):725-8.

6. Alexopoulos GS, Wilkins V, Marino P, Kanellopoulos D, Reding M, Sirey A, et al. Ecosystem focused therapy in post stroke depression: A preliminary study. Int J Geriatr Psychiatry. 2012;27(10):1053-60.

7. Parke HL, Epiphaniou E, Pearce G, Taylor SJC, Sheikh A, Griffiths CJ, et al. Self-Management Support Interventions for Stroke Survivors: A Systematic Meta-Review. PLoS One. 2015;10(7):e0131448.

8. Dossa A, Glickman ME, Berlowitz D. Association between mental health conditions and rehospitalization mortality and functional outcomes in patients with stroke following inpatient rehabilitation. BMC Health Serv Res. 2011;11(1):1-10.

9. Pustokhanova L, Morozova E. Cognitive impairment and hypothymia in post stroke patients. J Neurol Sci. 2013;325(1-2):43-5.

10. Pez-liria RL, Vega-ramirez FA, Rocamora-perez P, Aguilar-parra M, Padillagongora D. Comparison of Two Post-Stroke Rehabilitation Programs: A Follow-Up Study among Primary versus Specialized Health Care. PLoS One. 2016;11(11):e0166242.

11. Loong CK, Kenneth NKC, Paulin ST. Post-stroke depression: Outcome following rehabilitation. Aust N Z J Psychiatry. 1995;29(4):609-14

12. Lennon $O$, Blake $C$. Cardiac rehabilitation adapted to transient ischaemic attack and stroke (CRAFTS): A randomised controlled trial. BMC Neurol. 2009;9:1-8.

13. Wichowicz HM, Puchalska L, Rybak-Korneluk AM, Gąsecki D, Wisniewska A. Application of Solution-Focused Brief Therapy (SFBT) in individuals after stroke. Brain Inj. 2017;31(11):1-6.

14. Andersen G, Vestergaard K, Lauritzen L. Effective Treatment of Poststroke Depression with the Selective Serotonin Reuptake Inhibitor Citalopram. Stroke. 1994;25(6):1099-104.

15. Jakobsen JC, Hansen JL, Storebo OJ, Simonsen E, Gluud C. The Effects of Cognitive Therapy Versus "Treatment as Usual" in Patients with Major Depressive Disorder. PLoS One. 2011;6(8):e22890.
16. Thomas SA, Coates E, Nair R, Lincoln NB, Cooper C, Palmer R, et al. Behavioural Activation Therapy for Depression after Stroke (BEADS): A study protocol for a feasibility randomised controlled pilot trial of a psychological intervention for post-stroke depression. Pilot Feasibility Stud. 2016;2(1):1-12.

17. Mitchell PH, Veith RC, Becker KJ, Buzaitis A, Cain KC, Fruin M, et al. Brief psychosocial-behavioral intervention with antidepressant reduces poststroke depression significantly more than usual care with antidepressant: Living well with stroke: randomized, controlled trial. Stroke. 2009;40(9):3073-8.

18. Kootker JA, Fasotti L, Rasquin SM, Heugten CMV, Geurts AC. The effectiveness of an augmented cognitive behavioural intervention for Post-stroke Depression with or without Anxiety (PSDA). BMC Neurol. 2012;12(1):51-9.

19. Eeden MV, Kootker JA, Evers SMAA, Heugten CMV, Geurts ACH. An economic evaluation of an augmented cognitive behavioural intervention vs. Computerized cognitive training for post-stroke depressive symptoms. BMC Neurol. 2015;15(1):266-78.

20. Alexopolous GS, Raue PJ, Kiosses DN, Mackin S, Kanellopolous D, McCulloch C, et al. Problem Solving Therapy and Supportive Therapy in Older Adults with Major Depression and Executive Dysfunction: Effect on Disability. Arch Gen Psychiatry. 2011;68(1):33-41.

21. Robinson RG, Jorge RE, Long J. Prevention of Post-Stroke Mortality Using Problem Solving Therapy or Escitalopram. Am J Geriatr Psychiatry. 2016;25(5):512-9.

22. Barker-Collo $S$, Krishnamurthi R, Witt E, Feigin V, Jones A, Mcpherson $\mathrm{K}$, et al. Improving adherence to secondary stroke prevention strategies through motivational interviewing: Randomized controlled trial. Stroke. 2015;46(12):3451-8.

23. Eum Y, Yim J. Literature and Art Therapy in Post-Stroke Psychological Disorders. Tohoku J Exp Med. 2015;235(1):17-23.

24. Kim DS, Park YG, Choi JH, Im SH, Jung KJ, Cha YA, et al. Effects of music therapy on mood in stroke patients. Yonsei Med J. 2011;52(6):977-81.

25. Altenmuller EO, Schlaug G. Neurologic music therapy: The beneficial effects of music making on Neurorehabilitation. Acoust Sci Technol. 2013;34(1):5-12.

26. Forsblom A, Laitinen S, Teppo S, Tervaniemi M. Therapeutic Role of Music Listening in Stroke Rehabilitation. Ann N Y Acad Sci. 2009;1169(1):426-30.

27. Jessica L, Arpan S, Donglin Y, Jessica R. Acute stroke, effect on mood and music therapy: A non-pharmacological intervention. J Neurol. 2017;88(16 Supplement):288.

28. Amengual JL, Rojo N, Veciana M, Marco-pallares J, Grau-sanchez J, Schneider S, et al. Sensorimotor Plasticity after Music-Supported Therapy in Chronic Stroke Patients Revealed by Transcranial Magnetic Stimulation. Plos One. 2013;8(4):e61883.

29. Schneider S, Schonle PW, Altenmuller E, Munte TF. Using musical instruments to improve motor skill recovery following a stroke. J Neurol. 2007;254(10):1339-46.

30. Beesley K, White JH, Alston MK, Sweetapple AL, Pollack M. Art after stroke: The qualitative experience of community dwelling stroke survivors in a group art programme. Disabil Rehabil. 2011;33(23-24):2346-55.

31. Obembe A, Mapayi B, Johnson O, Agunbiade T, Pt BMR, Emechete A. Community reintegration in stroke survivors: Relationship with motor function and depression. Hong Kong Physiother J. 2013;31(2):69-74.

32. Block VAJ, Pitsch E, Tahir P, Cree BAC, Allen DD, Gelfand JM. Remote Physical Activity Monitoring in Neurological Disease: A Systematic Review. PLos One. 2016;11(4):e0154335.

33. Floel A, Werner C, Grittner U, Hesse S, Jobges M, Knauss J, et al. Physical fitness training in Subacute Stroke (PHYS-STROKE)-study protocol for a randomised controlled trial. Trials. 2014;15(1):1-12.

34. Bonner NS, Halloran PDO, Bernhardt J, Cumming TB. Developing the Stroke Exercise Preference Inventory (SEPI). PLoS One. 2016;11(10):e0164120.

35. Barak S, Hutzler Y, Dubnov-Razi G. Physical exercise after stroke: Effects, recommendations and barriers. Harefuah. 2016;155(6):378-83.

36. Plow M, Moore SM, Kirwan JP, Frost F, Katzan I, Jaeger S, et al. Randomized controlled pilot study of a System CHANGE TM weight management intervention in stroke survivors: Rationale and protocol. Trials. 2013;14:1-7.

37. Singh DKA, Azlin N, Nordin NAM, Aziz NAA, Lim KB, Soh LC. Effects of substituting a portion of standard physiotherapy time with virtual reality games among community-dwelling stroke survivors. BMC Neurol. 2013;13(1):199206. 
38. Prathikanti S, Rivera R, Cochran A, Tungol JG, Fayazmanesh N, Weinmann E. Treating major depression with yoga: A prospective, randomized, controlled pilot trial. PLoS One. 2017;12(3):e0173869.

39. Chan W, Immink MA, Hillier S. Yoga and exercise for symptoms of depression and anxiety in people with post stroke disability: A randomized, controlled pilot trial. Altern Ther Health Med. 2012;18(3):34-43.

40. Kawakita K, Okada K. Acupuncture therapy: Mechanism of action, efficacy and safety: A potential intervention for psychogenic disorders. Bio Psycho Social Medicine. 2014;8(1):4-10.

41. Man S, Hung BHB, Ng RMK, Yu X, Cheung H, Fung MPM, et al. A pilot controlled trial of a combination of dense cranial electroacupuncture stimulation and body acupuncture for post-stroke depression. BMC Complement Altern Med. 2014;14(1):255-63.

42. Guang-cai Z, Wen-bin F, Neng-gui X, Jian-hua L, Xiao-ping Z, Zhao-hui $L$, et al. Meta analysis of the curative effect of acupuncture on post-stroke depression. J Tradit Chinese Med. 2012;32(1):6-11.

43. Menghan L, Bo Z, Zhihong M, Tao S, Yuhui H, Hong Z, et al. Effect of Tiaoshen Kaiqiao acupuncture in the treatment of ischemic post-stroke depression: $\mathrm{A}$ randomized controlled trial. J Tradit Chinese Med. 2017;37(2):171-8.

44. Huang J, Lin Z, Wang Q, Liu F, Liu J, Fang Y, et al. The effect of a therapeutic regimen of Traditional Chinese Medicine rehabilitation for post-stroke cognitive impairment: study protocol for a randomized controlled trial. Trials. 2015;16:272-83.

45. Prokopenko SV, Mozheyko EY, Petrova MM, Koryagina TD, Kaskaeva DS, Chernykh TV, et al. Correction of post-stroke cognitive impairments using computer programs. J Neurol Sci. 2013;325(1-2):148-53.

46. Simblett SK, Yates M, Wagner AP, Watson P, Gracey F, Ring $\mathrm{H}$, et al. Computerized Cognitive Behavioral Therapy to Treat Emotional Distress
After Stroke: A Feasibility Randomized Controlled Trial. JMIR Ment Health. 2017;4(2):e16

47. Harmandayan M, Romanowicz M, Sola C. Successful use of ECT in poststroke depression. Gen Hosp Psychiatry. 2012;34(1):102.e5-6.

48. Shen X, Liu M, Cheng Y, Jia C, Pan X, Gou Q, et al. Repetitive transcranial magnetic stimulation for the treatment of post-stroke depression: A systematic review and meta-analysis of randomized controlled clinical trials. J Affect Disord. 2016;211:65-74.

49. Brunoni AR, Boggio PS, DeRaedt R, Bensenor IM, Lotufo PA, Namur V, et al. Cognitive control therapy and transcranial direct current stimulation for depression: A randomized, double-blinded, controlled trial. J Affect Disord. 2014;162:43-9.

50. Gu SY, Chang MC. The Effects of $10-\mathrm{Hz}$ Repetitive Transcranial Magnetic Stimulation on Depression in Chronic Stroke Patients. Brain Stimul. 2017;10(2):270-4.

51. Gray RJ, Myint PK, Elender F, Barton G, Pfeil M, Price G, et al. A Depression Recognition and Treatment package for families living with Stroke (DepReTStroke): Study protocol for a randomised controlled trial. Trials. 2011;12:1-8.

52. Lambercy O, Dovat L, Yun H, Wee SK, Kuah CW, Chua KS, et al. Effects of a robot-assisted training of grasp and pronation/supination in chronic stroke: A pilot study. J Neuroeng Rehabil. 2011;8:63-74.

53. Byl NN. Mobility training using a bionic knee orthosis in patients in a poststroke, chronic state: A case series. J Med Case Rep. 2012;6:1-5.

54. Linder SM, Rosenfeldt AB, Bay RC, Sahu K, Wolf SL, Alberts JL. Improving Quality of Life and Depression after Stroke Through Telerehabilitation. Am J Occup Ther. 2015;69(2):1-10.

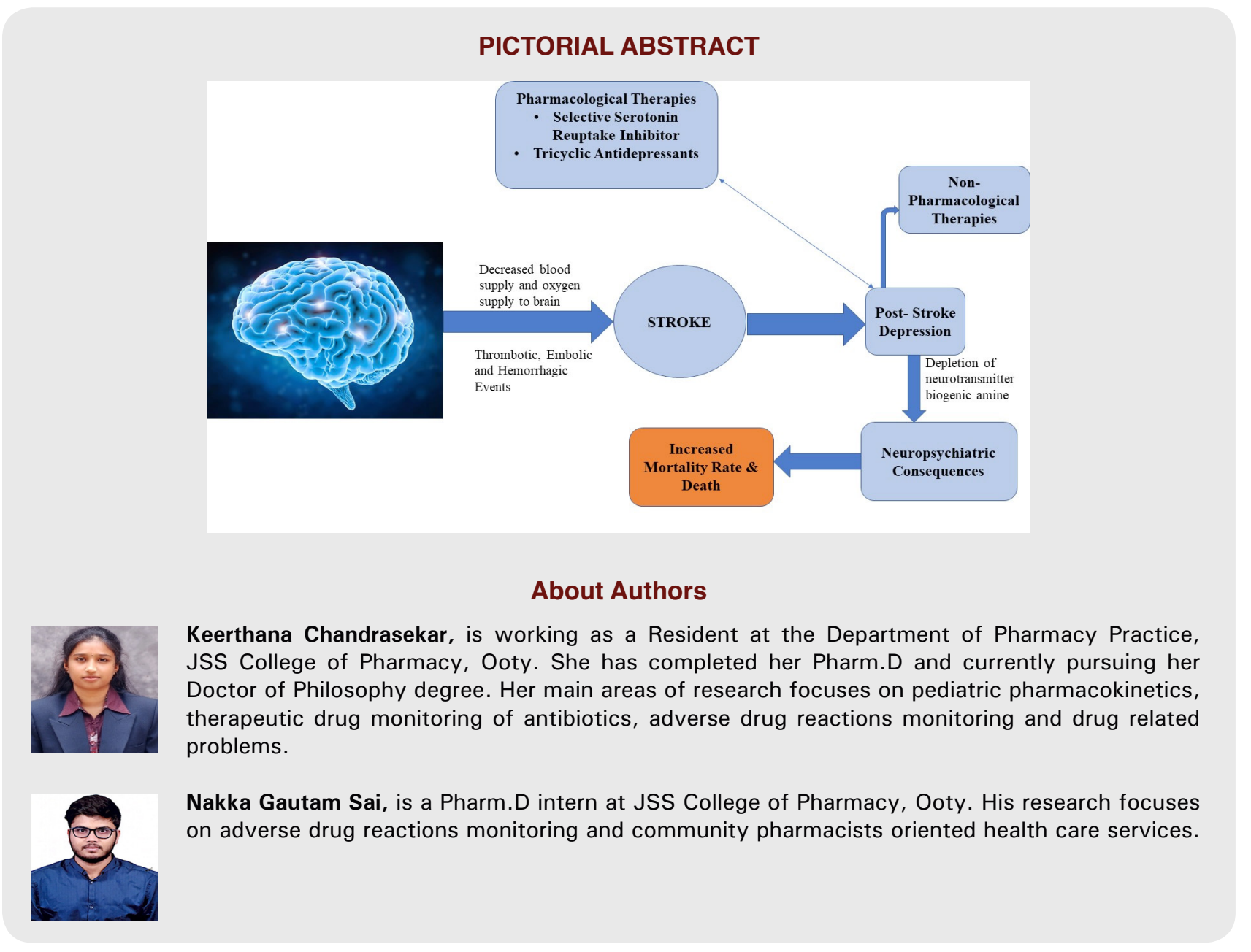



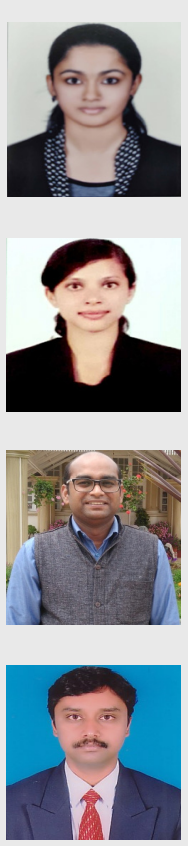

Princy Sabu John, is a Pharm.D intern at JSS College of Pharmacy, Ooty. Her research area focuses on drug related problems, adverse drug reactions monitoring in cardiovascular diseases, respiratory diseases and dose calculations in paediatrics.

Sruthi Ninan, is a Pharm.D intern at JSS College of Pharmacy, Ooty. Her research area focuses on drug related problems, adverse drug reactions monitoring in cardiovascular diseases and dose calculations in paediatrics.

Raja Durai, is working as a Assistant Professor at the Department of Pharmacy Practice, JSS College of Pharmacy, Ooty. His doctoral research focused on Diabetes Mellitus. His main areas of research focuses on developing application related to drug and poison information centre.

Dr. Sivasankaran Ponnusankar, is working as Professor and Head at the Department of Pharmacy Practice, JSS College of Pharmacy, Ooty. He is involved in developing clinical pharmacy practice/ services and education at Govt. District Headquarters Hospital, Ooty for the last 23 years. His research area of interest is Health Economics Outcome Research (HEOR) and Social, Preventive and Administrative Pharmacy.

Cite this article: Chandrasekar K, Sai NG, John PS, Ninan S, Durai R, Ponnusankar S. Emerging Non-Pharmacological Therapies for Post-stroke Depression and its Future Aspects: A Review. Indian J of Pharmaceutical Education and Research. 2020;54(1):1-7. 